\title{
Fluid Flow and Heat Transfer over a Permeable Stretching Cylinder
}

\author{
K. Vajravelu ${ }^{1}$, K. V. Prasad ${ }^{2 \dagger}$, S. R. Santhi ${ }^{3}$ and V. Umesh ${ }^{4}$ \\ ${ }^{I}$ Department of Mathematics; Department of Mechanical, Material and Aerospace Engineering; University of \\ Central Florida, Orlando, Florida 32816, USA \\ ${ }^{2}$ Department of Mathematics, VSK University, Bellary, Karnataka, India \\ ${ }^{3}$ Department of Mathematics, Cambridge Institute of Technology, Bangalore, India. \\ ${ }^{4}$ Department of Civil Engineering, SJB Institute of Technology, Bangalore, Karnataka India \\ †Corresponding Author Email: prasadkv2000@yahoo.co.in
}

(Received December 20, 2012; accepted January 23, 2013)

\begin{abstract}
In this paper, we analyze the effects of thermo-physical properties on the axisymmetric flow of a viscous fluid induced by a stretching cylinder in the presence of internal heat generation/absorption. It is assumed that the cylinder is stretched in the axial direction with a linear velocity and the surface temperature of the cylinder is subjected to vary linearly. Here, the temperature dependent thermo-physical properties namely, the fluid viscosity and the fluid thermal conductivity are respectively assumed to vary as an inverse function of the temperature and a linear function of the temperature. The governing system of partial differential equations is converted into a system of coupled non-linear ordinary differential equations with variable coefficients. The resulting system is solved numerically using a second order finite difference scheme known as the Keller-box method. The governing equations of the problem show that the flow and heat transfer characteristics depend on six parameters, namely the curvature parameter, fluid viscosity parameter, injection/suction parameter, variable thermal conductivity parameter, heat source/sink parameter and the Prandtl number. The numerical values obtained for the velocity, temperature, skin friction, and the Nusselt number are presented through graphs and tables for several sets of values of the pertinent parameters. The results obtained for the flow and heat transfer characteristics reveal many interesting behaviors that warrant further study on the axisymmetric flow phenomena. Comparisons with the available results in the literature are presented as special cases.
\end{abstract}

Keywords: Thermo-physical properties, Fluid flow, Heat transfer, Permeable cylinder, Boundary layer flow.

\section{NOMENCLATURE}

$\begin{array}{ll}A, c & \text { constants } \\ a & \text { radius of the cylinder } \\ b & \text { stretching rate } \\ c_{p} & \text { specific heat at constant pressure } \\ C_{f} & \text { skin friction coefficient } \\ f & \text { dimensionless stream function } \\ f_{w} & \text { suction/injection parameter } \\ K(T) & \text { temperature-dependent thermal } \\ & \text { conductivity } \\ k_{w} & \text { thermal conductivity at the wall } \\ k_{\infty} & \text { conductivity of the fluid away from the } \\ & \text { sheet } \\ l & \text { reference length } \\ N u_{x} & \text { local Nusselt number } \\ P r & \text { Prandtl number } \\ Q & \text { dimensional heat generation or absorption }\end{array}$

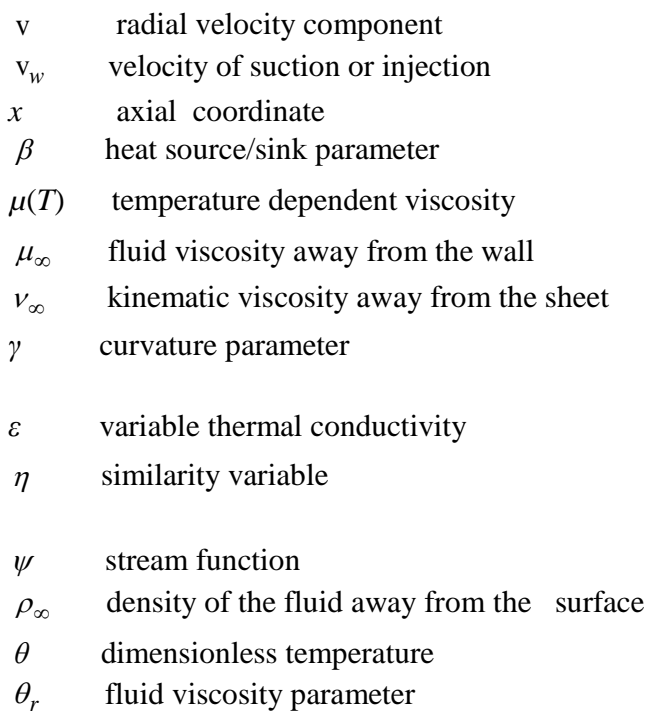




\author{
$r \quad$ radial coordinate \\ $\mathrm{Re}_{x} \quad$ local Reynolds number \\ $T \quad$ fluid temperature \\ $T_{w}(x)$ temperature of the stretching surface \\ $T_{\infty} \quad$ ambient temperature \\ $T_{r} \quad$ reference state \\ $U_{w}(x) \quad$ stretching velocity \\ $u \quad$ axial velocity component
}

\section{INTRODUCTION}

The study of two-dimensional viscous fluid flow from a permeable stretching surface is not only applicable to the elongation of bubbles and pseudopods but is particularly important in extrusion processes; such as metal and polymer extrusion, continuous casting, glass fiber production, manufacturing of plastic and rubber sheets, cable coating, crystal growing, spinning of filaments, wire drawing, etc. Also, suction or injection of a fluid through an elastic sheet finds applications in many engineering processes such as in the design of thrust bearing and radial diffusers, and thermal oil recovery. Furthermore, suction is applied to chemical processes to remove reactants; whereas blowing is used to add reactants, cool the surface, prevent corrosion or scaling and reduce drag. In general, suction tends to increase the skin friction and heat transfer coefficients; whereas injection acts quite the opposite way. In all these cases, the quality of final product depends on the rate of heat transfer at the stretching surface. The investigation of drag and heat transfer in such situations belongs to a special class of problems in which the boundary layer is quite different from that observed in flows over static surfaces. In view of these applications, Crane (1970) first obtained an elegant analytical solution to the boundary layer flow induced by a stretching surface in a quiescent fluid. Crane's (1970) work was subsequently extended to Newtonian and non-Newtonian fluids under different situations: To mention a few, Rajagopal et al. (1984), Grubka and Bobba (1985), Vajravelu (1994), Datti et al. (2004), Cortell (2005), Prasad et al. (2009), Gupta and Gupta (1977), Chen and Char (1988), Erickson et al. (1966).

All the above investigators restricted their analyses to flow and heat transfer problems over a stretching sheet. But not much has been done for the more intricate problem of the axisymmetric flow due to a stretching cylinder. Flow over a cylinder is considered to be two dimensional when the radius of the cylinder is large compared to the boundary layer thickness. On the other hand, for a thin cylinder, the radius may be of the same order as that of the boundary layer thickness. Therefore, the flow may be considered as axisymmetric instead of two-dimensional. In this case, the governing equations contain the transverse curvature term which may affect the velocity and the temperature fields. The effect of the transverse curvature is important in certain technological applications such as hot rolling, wire or fiber drawing where accurate prediction of flow and heat transfer is required and thick boundary layers can $\begin{array}{ll}\delta & \text { thermal property of the fluid } \\ \tau_{w} & \text { shear stress }\end{array}$

\section{Subscript}

$w \quad$ condition at the stretching surface

$\infty \quad$ condition at infinity

Superscript

' differentiation with respect to $\eta$

exist on slender or near slender bodies. In view of this, Crane (1975) studied the boundary layer flow due to a stretching cylinder. Wang (1988) extended the work of Crane (1975) to study the flow of a viscous fluid at a stretching hollow cylinder in an ambient fluid at rest. Pop et al. (1990) investigated the boundary layer flow past a moving longitudinal cylinder in a non-Newtonian power-law fluid at rest. Bachok and Ishak (2010) analyzed the effects of the governing parameters on the flow and heat transfer over a horizontal cylinder with prescribed surface heat flux.

In the above studies, the thermo-physical properties of the fluids are assumed to be constants. However, it is well known that, these properties change with temperature (Herwig and Wickern, 1986; Lai and Kulacki, 1990), especially the fluid viscosity and the thermal conductivity. For lubricating fluids, heat generated by internal friction and the corresponding rise in the temperature affects the physical properties of the fluid, and the properties of the fluid are no longer to be assumed constants. The increase in temperature leads to an increase in the transport phenomenon and therefore changes the physical properties across the thermal boundary layer, which affects the heat transfer at the wall. Therefore, to predict the flow and heat transfer rates, it is necessary to take into account the variable fluid properties. Available literature on the variable fluid properties (Ali, 2006; Hassanien, 1997; Abel et al., 2002; Prasad et al., 2010) shows that not much work has been carried out for the flow and heat transfer over a stretching cylinder.

Motivated by these applications, in this article, we analyze the effects of the variable fluid properties on the flow and heat transfer induced by a stretching permeable cylinder. In addition to this, we also consider the situations of endothermic/exothermic chemical reactions by including the effects of internal heat generation/absorption in the energy equation. Due to the influence of the transverse curvature, internal heat generation/absorption, and the temperature dependent fluid properties, the momentum and energy equations are coupled and highly non-linear. These partial differential equations are reduced to a system of coupled non-linear ordinary differential equations with variable coefficients. These equations are solved numerically by a finite difference scheme for different values of the parameters. The effects of the governing parameters on the velocity and temperature fields as well as the skin friction coefficient and the Nusselt number are presented in graphical and tabular forms. 
We believe that the results presented here will provide useful information for application and complement the results in the previous studies.

\section{MATHEMATICAL FORMULATION}

Let us consider the exterior viscous, incompressible and electrically conducting fluid flow due to the extrusion of a long impermeable hollow horizontal cylinder with radius $a$. The $x$-axis is measured along the axis of the cylinder from the lower stagnation point and $r$-axis is measured in the radial direction (see Fig.1).

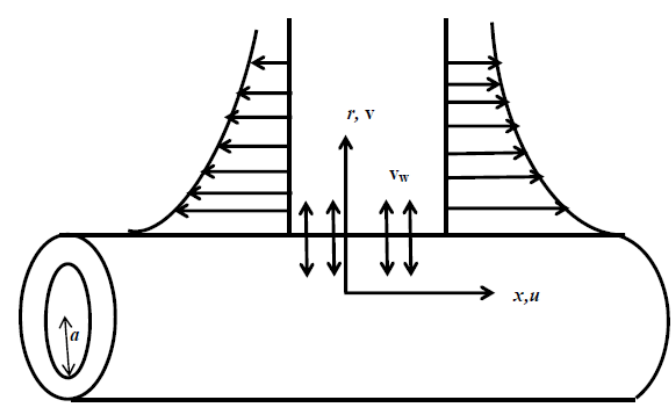

Fig. 1. Physical model and coordinate system

The horizontal cylinder is assumed to have linear stretching velocity $U_{w}(x)$ and surface temperature $T_{w}(x)$, which are of the form $U_{w}(x)=b(x / l)$ and $T_{w}(x)=c(x / l)$, where $b$ is the stretching rate, $c$ is the constant and $l$ is the reference length. All over the cylinder surface, the fluid is sucked out or ejected in with a constant radial velocity $\mathrm{v}_{\mathrm{w}}$. The thermophysical fluid properties are assumed to be isotropic and constant, except for the fluid viscosity and the thermal conductivity, which are assumed to vary as a function of temperature in the following forms

$\mu(T)=\frac{\mu_{\infty}}{\left[1+\delta\left(T-T_{\infty}\right)\right]}$

$k(T)=k_{\infty}\left(1+\varepsilon \frac{T-T_{\infty}}{\Delta T}\right)$

where $\mu(T)$ and $k(T)$ are respectively, the temperature dependent fluid viscosity and the fluid thermal conductivity. Here, $\mu_{\infty}$ and $k_{\infty}$ are respectively, the fluid viscosity and thermal conductivity far away from the surface. Further, $\varepsilon=\left(k_{w}-k_{\infty}\right) / k_{\infty}$ is a small parameter known as the variable thermal conductivity parameter, $\delta$ is a thermal property of the fluid, $\Delta T=T_{w}-T_{\infty}$, is the temperature difference, and $T_{w}$ and $k_{w}$ are respectively the temperature and thermal conductivity at the surface. For convenience, Eq. (1) can be rewritten as

$\mu(T)=\frac{1}{A\left(T-T_{r}\right)}$

where

$$
A=\frac{\delta}{\mu_{\infty}} \text { and } T_{r}=T_{\infty}-\frac{1}{\delta}
$$

In the above relation (4), both $A$ and $T_{r}$ are constant and their values depend on the reference state: In general, $A<0$ for a liquid and $A>0$ for gases. Let $\theta_{r}$ (fluid viscosity parameter) be the constant which is defined by

$\theta_{r}=\frac{T_{r}-T_{\infty}}{\Delta T}=-\frac{1}{\delta \Delta T}$

It is worth mentioning here that for $\delta \rightarrow 0$, i.e, $\mu(T)=\mu_{\infty}$ (constant), $\theta_{r} \rightarrow \infty$. It is also important to note that $\theta_{r}$ is negative for liquids and positive for gases. This is due to the fact that viscosity of a liquid usually decreases with increasing temperature; while it increases for gases. The most common working fluids found in engineering applications are air and water. To demonstrate further the appropriateness of Eq. (4), correlations between viscosity and temperature for air and water are given below

For $\quad$ air, $\quad \frac{1}{\mu}=-123.2(T-742.6)$ based

on

$T_{0}=293 K\left(20^{\circ} \mathrm{C}\right)$

for water, $\frac{1}{\mu}=-29.83(T-258.6)$ based on

$T_{0}=288 K\left(15^{0} \mathrm{C}\right)$

The data used for these correlations are taken from Weast (1986), While Eq. (6) is good up to an error within $1.2 \%$ to the temperature difference from $278 \mathrm{~K}$ $\left(5^{0} \mathrm{C}\right)$ to $373 K\left(100^{\circ} \mathrm{C}\right)$, Eq. (7) is good to an error within $5.8 \%$ to the temperature difference from $283 \mathrm{~K}$ $\left(10^{\circ} \mathrm{C}\right)$ to $373 K\left(100^{\circ} \mathrm{C}\right)$. Hence, the reference temperature selected here for the correlations are very useful for most applications. Under these assumptions, the boundary layer equations governing the flow and heat transfer in the presence of variable fluid properties are

$$
\begin{aligned}
& \frac{\partial}{\partial x}(r u)+\frac{\partial}{\partial r}(r \mathrm{v})=0 \\
& u \frac{\partial u}{\partial x}+\mathrm{v} \frac{\partial u}{\partial r}=\frac{1}{\rho_{\infty} r} \frac{\partial}{\partial r}\left(\mu(T) r \frac{\partial u}{\partial r}\right)
\end{aligned}
$$

$$
\begin{array}{r}
\left(u \frac{\partial T}{\partial x}+\mathrm{v} \frac{\partial T}{\partial r}\right)=\frac{1}{\rho_{\infty} c_{p} r} \frac{\partial}{\partial r}\left(k(T) r \frac{\partial T}{\partial r}\right) \\
+\frac{Q}{\rho_{\infty} c_{p}}\left(T-T_{\infty}\right)
\end{array}
$$

Here $(x, r)$ are cylindrical coordinates measured along the axis of the cylinder and in the radial direction, $(u, \mathrm{v})$ are the velocity components measured along 
the $x$ and $r$ directions respectively. $\rho_{\infty}$ is the density, $T$ is the temperature of the fluid in the boundary layer, $c_{p}$ is the specific heat at constant pressure, and $T_{\infty}$ is the ambient temperature. The last term containing $Q$ in Eq. (10) represents the temperature-dependent volumetric rate of heat addition when $Q>0$ and heat absorption when $Q<0$. These deal with the situation of exothermic and endothermic chemical reactions, respectively. The appropriate boundary conditions are

$u=U_{w}(x), \mathrm{v}= \pm \mathrm{v}_{\mathrm{w}}, \quad T=T_{w}(x)$ at $r=a$

$u \rightarrow 0, T \rightarrow T_{\infty}$ as $r \rightarrow \infty$

The momentum and energy equations can be transformed into ordinary differential equations by the following transformation (Mahamood and Merkin, 1988; Ishak and Nazar, 2009)

$$
\begin{aligned}
& \eta=\frac{r^{2}-a^{2}}{2 a}\left(U_{w} / v_{\infty} x\right)^{\frac{1}{2}}, \\
& \psi=\left(U_{w} v_{\infty} x\right)^{\frac{1}{2}} a f(\eta), \theta(\eta)=\frac{T-T_{\infty}}{T_{w}-T_{\infty}}
\end{aligned}
$$

here $\eta$ is the similarity variable, $f$ and $\theta$ are dimensionless quantities and $v_{\infty}$ is the kinematic viscosity. The continuity equation is being satisfied by the steam function $\psi: u=r^{-1} \partial \psi / \partial r$ and $\mathrm{v}=-r^{-1} \frac{\partial \psi}{\partial x}$

Using Eq. (12), we obtain the velocity components as

$$
u=U_{w} f^{\prime}, \quad \mathrm{v}=-\frac{a}{r}\left(\frac{v_{\infty} b}{l}\right)^{1 / 2} f
$$

where a prime denotes differentiation with respect to $\eta$. By defining $\eta$ in this form, the boundary condition at $r=a$ reduces to the boundary condition at $\eta=0$, which is more convenient for numerical computation. Substituting Eq. (12) into Eq. (9) and (10), we obtain the following ordinary differential equation:

$$
\begin{aligned}
& \left(\left(1-\frac{\theta}{\theta_{r}}\right)^{-1}(1+2 \eta \gamma) f^{\prime \prime}\right)^{\prime}-\left(f^{\prime}\right)^{2}+f f^{\prime \prime}=0 \\
& (1+2 \eta \gamma)\left((1+\varepsilon \theta) \theta^{\prime}\right)^{\prime}+2(1+\varepsilon \theta) \gamma \theta^{\prime} \\
& -\operatorname{Pr}\left(f^{\prime} \theta-f \theta^{\prime}\right)+\operatorname{Pr} \beta \theta=0
\end{aligned}
$$

subjected to the boundary conditions

$$
\begin{aligned}
& f(0)=f_{w}, f^{\prime}(0)=1, \theta(0)=1 \\
& f^{\prime} \rightarrow 0, \quad \theta \rightarrow 0 \text { as } \eta \rightarrow \infty .
\end{aligned}
$$

The parameters $f_{w}, \gamma, \operatorname{Pr}$ and $\beta$ are the suction/ injection parameter, the transverse curvature, the
Prandtl number, and the heat source/sink parameter, respectively: They are defined by

$f_{w}= \pm \mathrm{v}_{w} \sqrt{l / v_{\infty} b}, \gamma=\sqrt{\frac{l v_{\infty}}{b a^{2}}}, P_{r}=\frac{v_{\infty}}{\alpha_{\infty}}, \beta=\frac{Q l}{b \rho_{\infty} c_{p}}$

Here, the parameter $f_{w}$ corresponds to suction when $f_{w}>0$; whereas $f_{w}<0$ corresponds to blowing. We noticed that in the absence of transverse curvature and suction/injection, Eqs. (13) and (14) reduce to those of Hassanien (1997), while in the absence of the thermophysical properties and no curvature, equations reduce to those of Gupta and Gupta (1977), Further, in the presence of curvature and no thermo-physical properties, the equations reduce to those of Ishak and Nazar (2009), Also, when the transverse curvature and the thermo-physical properties are absent, the analytical solutions of the equations with the boundary conditions represent the flow and heat transfer results for the Newtonian fluid: Our results agree very well with the results of Crane (1970), and Grubka and Bobba (1985). The physical quantities of interest are the skin friction coefficient $C_{f}$ and the local Nusselt number $N u_{x}$ which are defined by

$$
C_{f}=\frac{\tau_{w}}{\rho_{\infty} U_{w}^{2} / 2}, N u_{x}=\frac{x q_{w}}{k_{\infty}\left(T_{w}-T_{\infty}\right)}
$$

where the surface shear stress and the surface heat flux are given by

$$
\tau_{w}=\mu\left(\frac{\partial u}{\partial r}\right)_{r=a}, q_{w}=-k_{\infty}\left(\frac{\partial T}{\partial r}\right)_{r=a}
$$

with $\mu_{\infty}$ and $k_{\infty}$ being respectively, the viscosity and the thermal conductivity of the fluid far from the surface. Using the similarity variables Eq. (12), we obtain

$\frac{1}{2} C_{f} \operatorname{Re}_{x}^{1 / 2}=f^{\prime \prime}(0)$, and $\frac{N u_{x}}{\operatorname{Re}_{x}^{1 / 2}}=-\theta^{\prime}(0)$

where $\operatorname{Re}_{x}=U_{w} x / \nu_{\infty}$ is the local Reynolds number.

\section{Numerical Procedures}

The system of coupled non-linear Eqs. (13) - (14) with variable coefficients subject to the boundary conditions (15) is solved numerically by an implicit finite difference scheme known as the Keller-box method (for details see Cebeci and Bradshaw, 1984; Keller,1992; Prasad et al., 2009). The axisymmetric flow and heat transfer at the permeable horizontal cylinder is controlled by the non-dimensional parameters, namely, the transverse curvature parameter, the fluid viscosity parameter, the injection parameter, the internal heat source/sink parameter, the Prandtl number and the variable thermal conductivity parameter. The main focus of the present study is to bring out the effects of these parameters through the numerical results for the skin friction and the wall temperature gradient. For 
numerical calculations, a uniform step size of $h=0.01$ is found to be satisfactory and the solutions are obtained within an error tolerance of $10^{-6}$. The step size $\Delta \eta$ and the position of the edge of the boundary layer $\eta_{\infty}$ are to be adjusted for different values of the governing parameters to maintain the accuracy. For brevity, the details of the solution procedure are not presented here. It is also important to note that the computational time for each set of input parametric values should be short. Because the physical domain in this problem is unbounded, whereas the computational domain has to be finite, we apply the far field boundary conditions for the similarity variable $\eta$ at finite value denoted by $\eta_{\max }$. We ran the bulk of computations with the value $\eta_{\max }=12$, which is sufficient to achieve the far field boundary conditions asymptotically, for all values of the parameters considered. The accuracy of the numerical scheme is validated by comparing the skin friction and the rate of heat transfer results with those available in the literature: These results agree very well (see Table 1 and 2).

Table 1 Comparison of $-f$ " $(0)$ for different values of $f_{w}$ when $\gamma=0, \quad \theta_{r} \rightarrow \infty$

\begin{tabular}{|c|c|c|c|c|c|c|c|c|c|}
\hline & $f_{w}=-1.0$ & $\begin{array}{c}f_{w}=- \\
0.5\end{array}$ & $\begin{array}{c}f_{w}=- \\
0.25\end{array}$ & $\begin{array}{c}f_{w}=- \\
0.1\end{array}$ & $f_{w}=0.0$ & $f_{w}=0.1$ & $\begin{array}{c}f_{w}=0.2 \\
5\end{array}$ & $f_{w}=0.5$ & $\begin{array}{c}f_{w}= \\
1.0\end{array}$ \\
\hline $\begin{array}{l}\text { Numerical } \\
\text { solution }\end{array}$ & 0.618063 & 0.780800 & 0.882798 & 0.951260 & 1.000180 & 1.051255 & 1.132786 & 1.280778 & 1.618034 \\
\hline $\begin{array}{l}\text { Analytical } \\
\text { solution }\end{array}$ & 0.618034 & 0.780780 & 0.882782 & 0.951250 & 1.000000 & 1.051249 & 1.132780 & 1.280780 & 1.618034 \\
\hline
\end{tabular}

Table 2 Comparison of $-\theta^{\prime}(0)$ for different values of $\operatorname{Pr}$ when $\gamma=0.0, \varepsilon=0.0, \beta=0.0$ and $\theta_{r} \rightarrow \infty$.

\begin{tabular}{|c|c|c|c|c|}
\hline $\operatorname{Pr}$ & $\begin{array}{c}\text { Grubkha and } \\
\text { Bobba (1985) }\end{array}$ & Ali (1994) & Ishak et al. (2009) & Present result \\
\hline 0.72 & 0.8086 & 0.8058 & 0.808631350 & 0.808631 \\
\hline 1.0 & 1.0000 & 0.9961 & 1.0000000000 & 1.000000 \\
\hline 3.0 & 1.9237 & 1.9144 & 1.923682595 & 1.923663 \\
\hline 10.0 & 3.7207 & 3.7006 & 3.720673901 & 3.720649 \\
\hline
\end{tabular}

\section{RESULTS AND DISCUSSION}

The influence of the temperature-dependent fluid properties and the transverse curvature on the axisymmetric flow and heat transfer at a horizontal permeable stretching cylinder is investigated numerically. Analytical solutions are obtained for the special case when $\theta_{r} \rightarrow \infty, \gamma=0, \beta=0$ and $\varepsilon=0$. The warranted numerical solution for the general case is obtained by using a second order finite difference scheme known as the Keller-box method. In order to have an understanding of the mathematical model, we present the numerical results, graphically for the horizontal velocity and the temperature field, respectively in Figs. 2(a) to 2(d) and in Figs. 3 to 6. The local skin friction $f^{\prime \prime}(0)$ and the dimensionless walltemperature gradient $\theta^{\prime}(0)$ are presented in Table 3 for different values of the pertinent parameters.

Figures 2(a) to 2(c) respectively, show the effects of $\operatorname{suction}\left(f_{w}>0\right), \quad$ impermeability $\left(f_{w}=0\right), \quad$ and blowing $\left(f_{w}<0\right)$ on the horizontal velocity $f^{\prime}(\eta)$ for different values of the fluid viscosity parameter $\theta_{r}$ and the transverse curvature parameter $\gamma$ with $\operatorname{Pr}=1.0$. From these figures it can be seen that $f^{\prime}(\eta)$ decreases asymptotically to zero as the variable $\eta$ increases. The effect of increasing values of the fluid viscosity parameter $\theta_{r}$ is to decrease the momentum boundary layer thickness. Also, for $-1<\theta_{r}<0$, the boundary layer thickness decrease and the horizontal velocity distribution asymptotically tend to zero (see Figs. 2(a) to 2(c)). This is due to the fact that, for a given fluid, when $\delta$ is fixed, smaller $\theta_{r}$ implies higher temperature difference between the wall and the ambient fluid. The results presented in this paper demonstrate clearly that $\theta_{r}$, the indicator of the variation of fluid viscosity with temperature, has a substantial effect on the horizontal velocity $f^{\prime}(\eta)$ and hence on the skin friction. This observation also holds for non-zero values of the transverse curvature parameter. The effect of increasing values of the transverse curvature parameter is to increase the horizontal velocity and thereby enhance the boundary layer thickness. This phenomenon is even true for all values of injection parameter. It can be seen that the suction reduces the horizontal boundary layer thickness whereas the blowing has the opposite effect on it. These results are consistent with the physical situation.

In Figs. 3 to 6, the numerical results for the temperature profiles $\theta(\eta)$ for several sets of values of the governing parameters are presented. The general trend is that the temperature distribution is unity at the surface and with the changes in the physical parameters tends asymptotically to zero in the free stream region. 

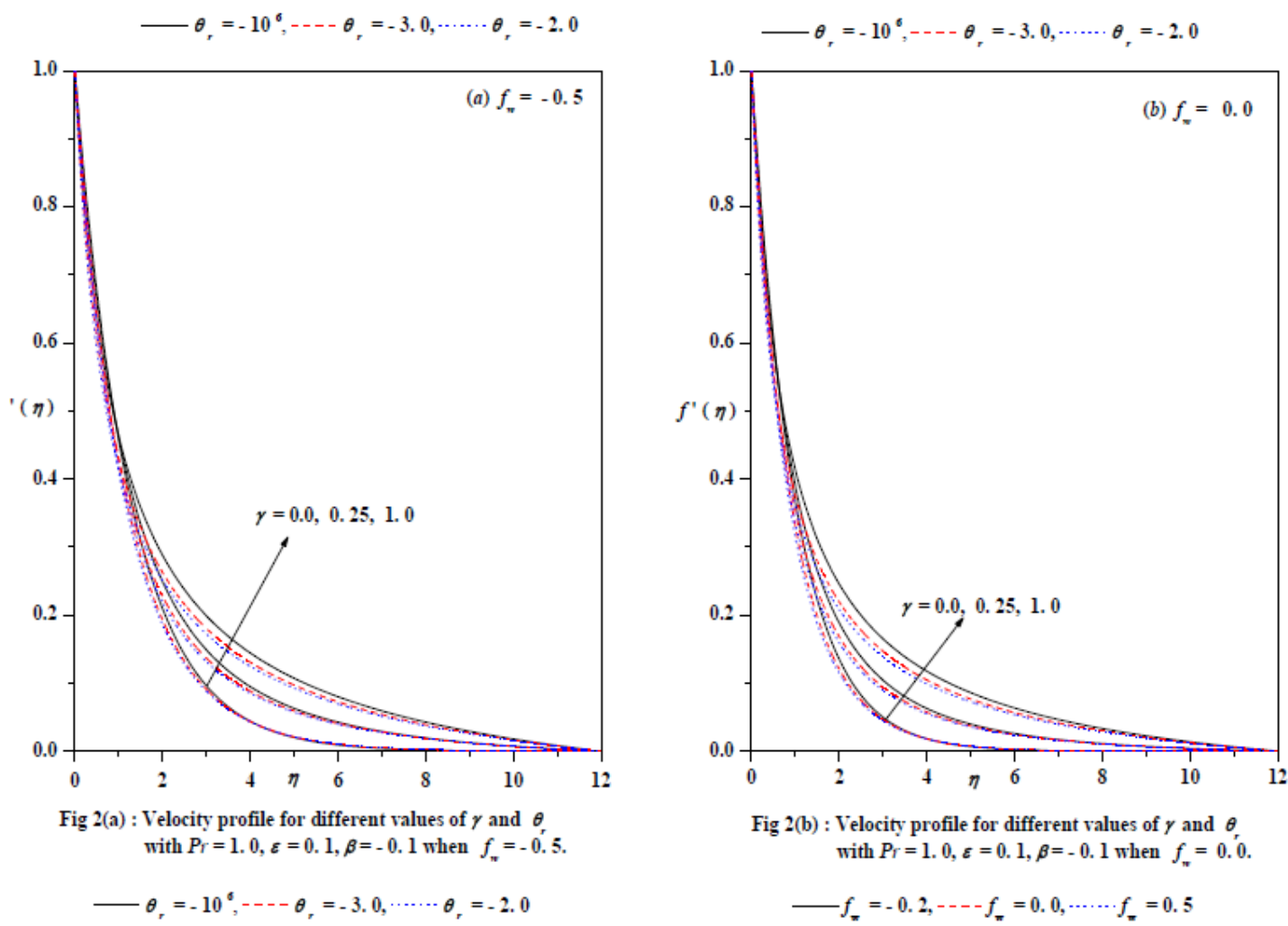

Fig 2(b) : Velocity profile for different values of $\gamma$ and $\theta$ with $P_{r}=1.0, \varepsilon=0.1, \beta=-0.1$ when $f_{n}=0.0$.
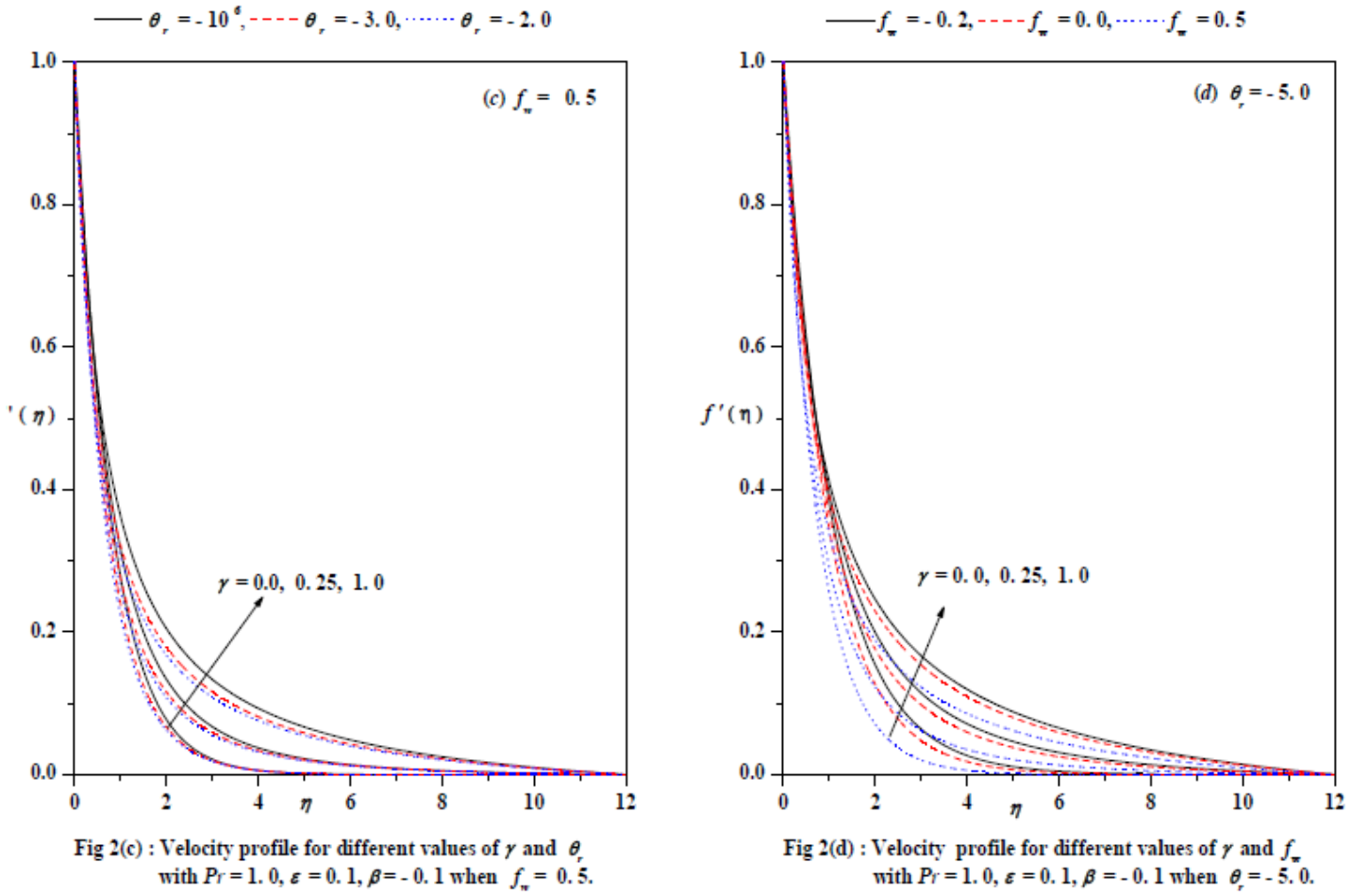

The effects of fluid viscosity parameter and the transverse curvature parameter on temperature profiles $\theta(\eta)$ in the boundary layer for all values of injection parameter namely, suction, impermeability and blowing cases are shown respectively in Figs. 3(a) to 3(c). From the Fig. 3(a), we observe that the effect of increasing values of the fluid viscosity parameter $\theta_{r}$ is to enhance the temperature. This is due to the fact that an increase

in the fluid viscosity parameter $\theta_{r}$ results in an increase in the thermal boundary layer thickness. This behavior is quite the opposite with non-zero values of the transverse curvature. The effect of increasing the values of the transverse curvature leads to decrease the thermal boundary thickness. This observation holds true for all values of the injection parameter. 

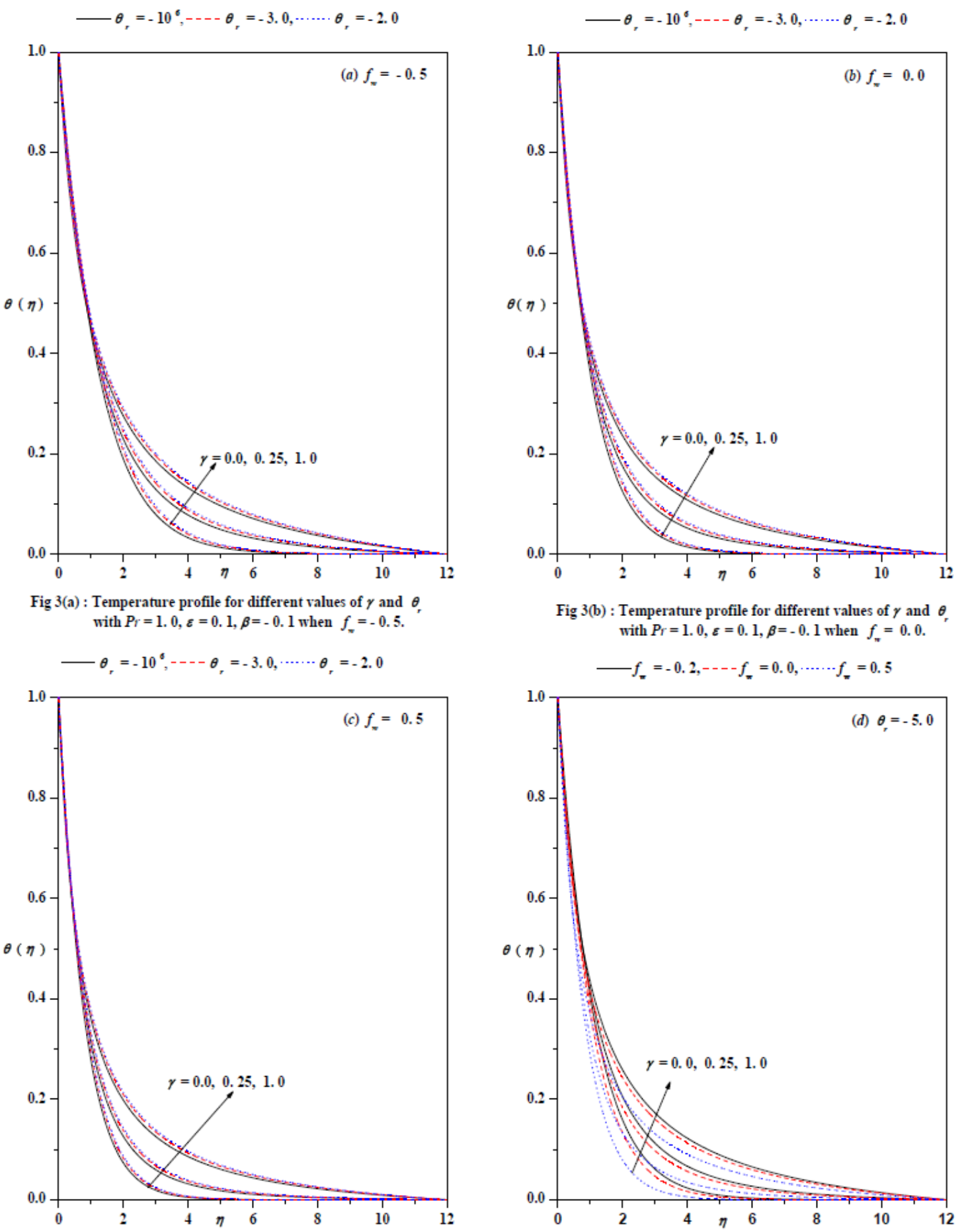

Fig 3(c) : Temperature profile for different values of $\gamma$ and $\theta_{r}$ with $P r=1.0, \varepsilon=0.1, \beta=-0.1$ when $f_{n}=0.5$.

From the graphical representation (Fig. 3(d)), we observe that an increase in $f_{w}$ leads to a decrease in the temperature and hence, the magnitude of the walltemperature gradient increases. This is due to the fact that the thermal boundary layer is thicker in the case of suction as compared to the case of impermeability: However, it is thinner in the case of blowing. Figures $4(\mathrm{a})$ and $4 \mathrm{~b}$ exhibit the temperature distribution $\theta(\eta)$ with $\eta$ for different values of the curvature

parameter and the heat source/sink parameter $\beta$ for both suction and blowing. From these graphs we observe that the temperature distribution is lower throughout the boundary layer for negative values of $\beta$ (heat sink) and higher for positive values of $\beta$ (heat source). Physically $\beta>0$ implies $T_{w}>T_{\infty}$ i.e. the supply of heat to the flow region from the wall. Similarity, $\beta<0$ implies $T_{w}<T_{\infty}$ i.e. the transfer of 
heat is from the fluid to the wall. The effect of increasing values of the heat source/sink parameter $\beta$ is to increase the temperature. This holds true for all the values of the transverse curvature. The effect of the variable thermal conductivity parameter $\varepsilon$ and the curvature parameter on the temperature for both suction and blowing parameters are shown graphically in Figs. 5 (a) and 5(b). These profiles demonstrate quite clearly that an increase in the value of $\varepsilon$ results in an increase in the temperature and hence the thermal boundary layer thickness increases as $\varepsilon$ increases. This is due to the fact that the assumption of temperature-dependent thermal conductivity causes a reduction in the magnitude of the transverse velocity by the quantity $\partial k(T) / \partial r$ :Seen from the energy Eq. (14). The variations of the temperature with $\eta$ for various values of the Prandtl number Pr are displayed in Figs. 6a and $6 \mathrm{~b}$ for both suction and blowing, respectively. These

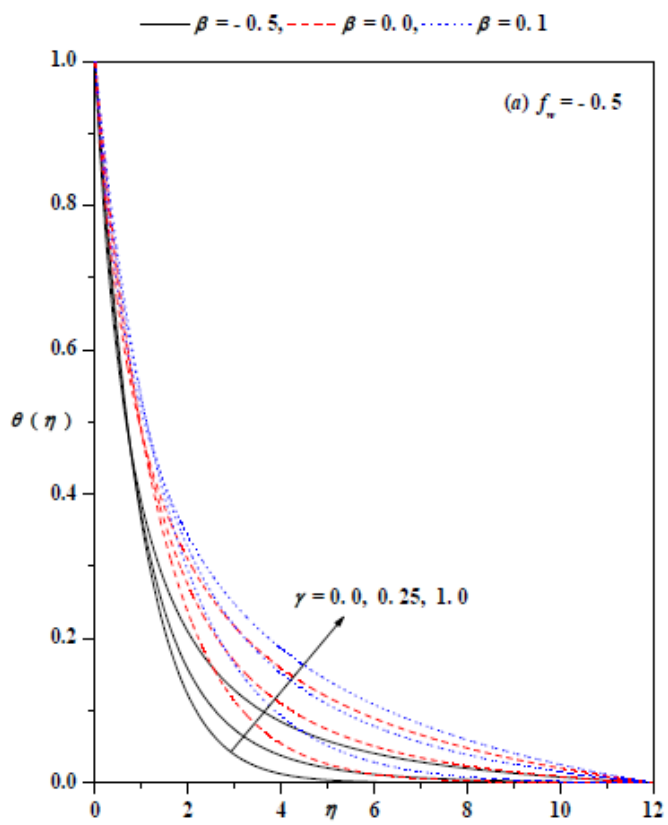

Fig 4(a) : Temperature profile for different values of $\gamma$ and $\beta$ with $P_{r}=1.0, \varepsilon=0.1, \theta_{r}=-5.0$ when $f_{v}=-0.5$. figures demonstrate that an increase in the Prandtl number $P r$ results in a decrease in the temperature distribution; and hence the thermal boundary layer thickness decrease.

The values of $f^{\prime \prime}(0)$ proportional to the local skin friction, and the wall temperature gradient $\theta^{\prime}(0)$ proportional to the local Nusselt number for different values of the pertinent parameters are recorded in Table 3. Analysis of the tabular data shows that the effect of the injection parameter and the variable viscosity parameter is to decrease the skin friction: But quite the opposite is true with the rate of heat transfer. However, the effects of the heat source/sink parameter and the variable thermal conductivity parameter are to enhance the rate of heat transfer. On the other hand, the effect of the Prandtl number and the transverse curvature is to decrease the wall-temperature gradient.

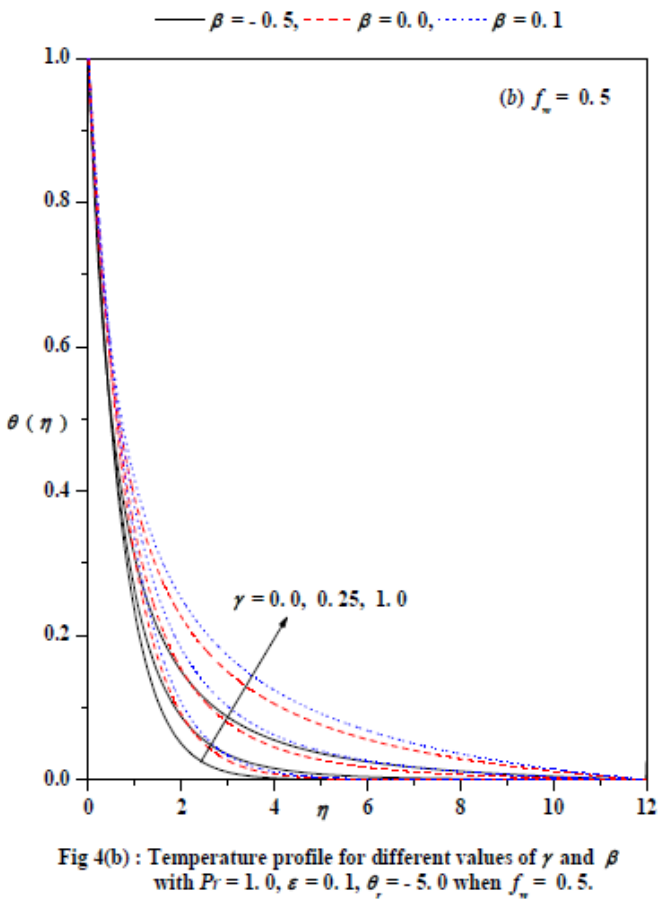

Table 3 Numerical values for the skin friction $-f^{\prime \prime}(0)$ and the wall-temperature gradient $-\theta^{\prime}(0)$ for different values of the physical parameters

\begin{tabular}{|c|c|c|c|c|c|c|c|c|c|c|c|c|c|c|}
\hline \multirow[b]{2}{*}{$\operatorname{Pr}$} & \multirow[b]{2}{*}{$\varepsilon$} & \multirow[b]{2}{*}{$\beta$} & \multirow[b]{2}{*}{$f_{w}$} & \multirow[b]{2}{*}{$\theta_{r}$} & \multicolumn{2}{|c|}{$\gamma=0$} & \multicolumn{2}{|c|}{$\gamma=0.25$} & \multicolumn{2}{|c|}{$\gamma=0.5$} & \multicolumn{2}{|c|}{$\gamma=0.75$} & \multicolumn{2}{|c|}{$\gamma=1.0$} \\
\hline & & & & & $-f^{\prime \prime}(0)$ & $-\theta^{\prime}(0)$ & $-f^{\prime \prime}(0)$ & $-\theta^{\prime}(0)$ & $-f^{\prime \prime}(0)$ & $-\theta^{\prime}(0)$ & $-f^{\prime \prime}(0)$ & $-\theta^{\prime}(0)$ & $-f^{\prime \prime}(0)$ & $-\theta^{\prime}(0)$ \\
\hline \multirow{4}{*}{1.0} & \multirow{4}{*}{0.1} & \multirow{4}{*}{-0.1} & \multirow{4}{*}{0.0} & $\infty$ & 1.00001 & 0.98940 & 1.09183 & 1.08233 & 1.18242 & 1.17129 & 1.27115 & 1.25745 & 1.35821 & 1.34151 \\
\hline & & & & -10.0 & 1.06119 & 0.98082 & 1.15670 & 1.07363 & 1.25101 & 1.16250 & 1.34344 & 1.24860 & 1.43416 & 1.33262 \\
\hline & & & & -3.0 & 1.18921 & 0.96251 & 1.29280 & 1.05513 & 1.39524 & 1.14384 & 1.49574 & 1.22984 & 1.59447 & 1.31380 \\
\hline & & & & -2.0 & 1.27668 & 0.95037 & 1.38816 & 1.04275 & 1.49839 & 1.13128 & 1.60656 & 1.21716 & 1.71282 & 1.30103 \\
\hline \multirow{3}{*}{1.0} & \multirow{3}{*}{0.1} & \multirow{3}{*}{-0.1} & -0.2 & \multirow{3}{*}{-5.0} & 1.00387 & 0.88978 & 1.10502 & 0.98382 & 1.20351 & 1.07307 & 1.29952 & 1.15924 & 1.39354 & 1.24319 \\
\hline & & & 0.0 & & 1.11823 & 0.97270 & 1.21713 & 1.06543 & 1.31486 & 1.15423 & 1.41069 & 1.24028 & 1.50480 & 1.32427 \\
\hline & & & 0.5 & & 1.45888 & 1.21741 & 1.54468 & 1.30086 & 1.63461 & 1.38446 & 1.72568 & 1.46730 & 1.81666 & 1.54917 \\
\hline \multirow{3}{*}{1.0} & \multirow{3}{*}{0.1} & -0.5 & \multirow{3}{*}{0.5} & \multirow{3}{*}{-5.0} & 1.46213 & 1.40406 & 1.54878 & 1.50060 & 1.63947 & 1.59278 & 1.73119 & 1.68151 & 1.82272 & 1.76758 \\
\hline & & 0.0 & & & 1.45785 & 1.16189 & 1.54325 & 1.23795 & 1.63291 & 1.31833 & 1.72378 & 1.39979 & 1.81460 & 1.48107 \\
\hline & & 0.1 & & & 1.45665 & 1.10032 & 1.54148 & 1.16382 & 1.63082 & 1.24103 & 1.72149 & 1.32211 & 1.81218 & 1.40380 \\
\hline \multirow{3}{*}{1.0} & 0.0 & \multirow{3}{*}{-0.1} & \multirow{3}{*}{0.5} & \multirow{3}{*}{-5.0} & 1.46023 & 1.31596 & 1.54623 & 1.40282 & 1.63635 & 1.48981 & 1.72761 & 1.57603 & 1.81875 & 1.66127 \\
\hline & 0.2 & & & & 1.45765 & 1.13437 & 1.54326 & 1.21499 & 1.63302 & 1.29582 & 1.72392 & 1.37588 & 1.81474 & 1.45496 \\
\hline & 0.4 & & & & 1.45547 & 1.00178 & 1.54075 & 1.07804 & 1.63019 & 1.15457 & 1.72080 & 1.23035 & 1.81134 & 1.30515 \\
\hline 1.0 & \multirow{3}{*}{0.1} & \multirow{3}{*}{-0.1} & \multirow{3}{*}{0.5} & \multirow{3}{*}{-5.0} & 1.45888 & 1.21741 & 1.54468 & 1.30086 & 1.63461 & 1.38446 & 1.72568 & 1.46730 & 1.81666 & 1.54917 \\
\hline 2.0 & & & & & 1.47135 & 2.01658 & 1.55869 & 2.09528 & 1.64999 & 2.17279 & 1.74222 & 2.24932 & 1.83419 & 2.32505 \\
\hline 3.0 & & & & & 1.47941 & 2.70835 & 1.56779 & 2.78399 & 1.66006 & 2.85881 & 1.75321 & 2.93298 & 1.84602 & 3.00656 \\
\hline
\end{tabular}




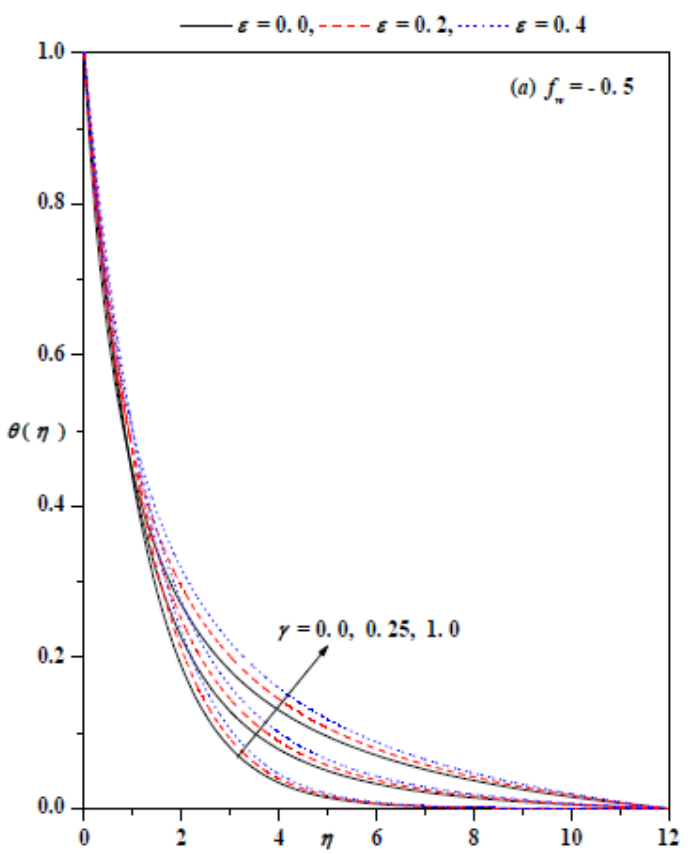

Fig 5 (a) : Temperature profile for different values of $\gamma$ and $\varepsilon$ with $P r=1.0, \beta=-0.1, \theta_{r}=-5.0$ when $f_{n}=-0.5$.

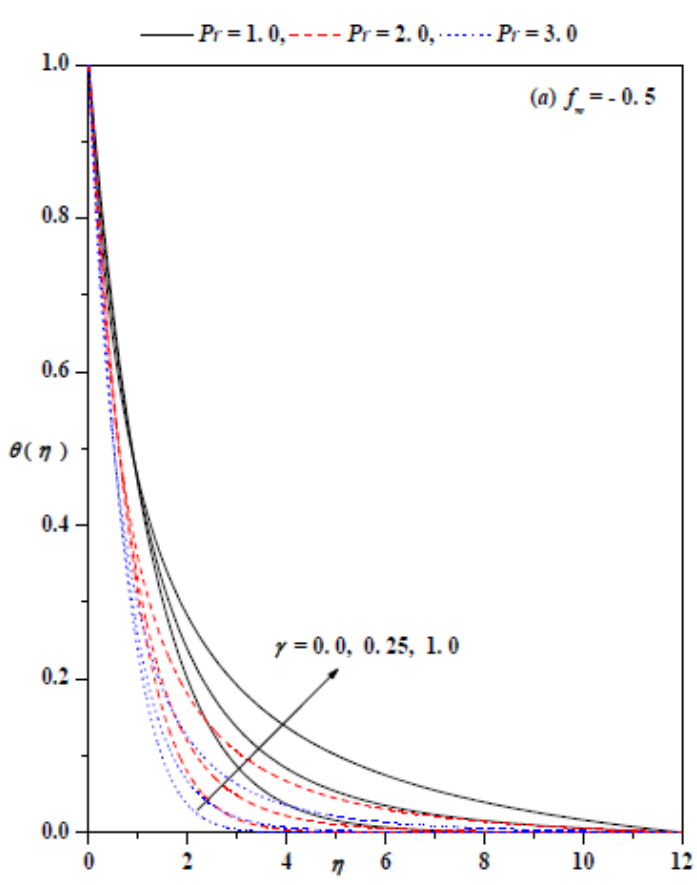

Fig 6 (a) : Temperature profile for different values of $\gamma$ and $P r$ with $\beta=-0.1, \varepsilon=0.1, \theta_{r}=-5.0$ when $f_{z}=-0.5$.

\section{CONCLUSiON}

Some of the interesting conclusions are as follows:

- In the presence of temperature-dependent thermophysical properties, the effect of increasing values of transverse curvature parameter is to increase the velocity and the thermal boundary layer thicknesses;

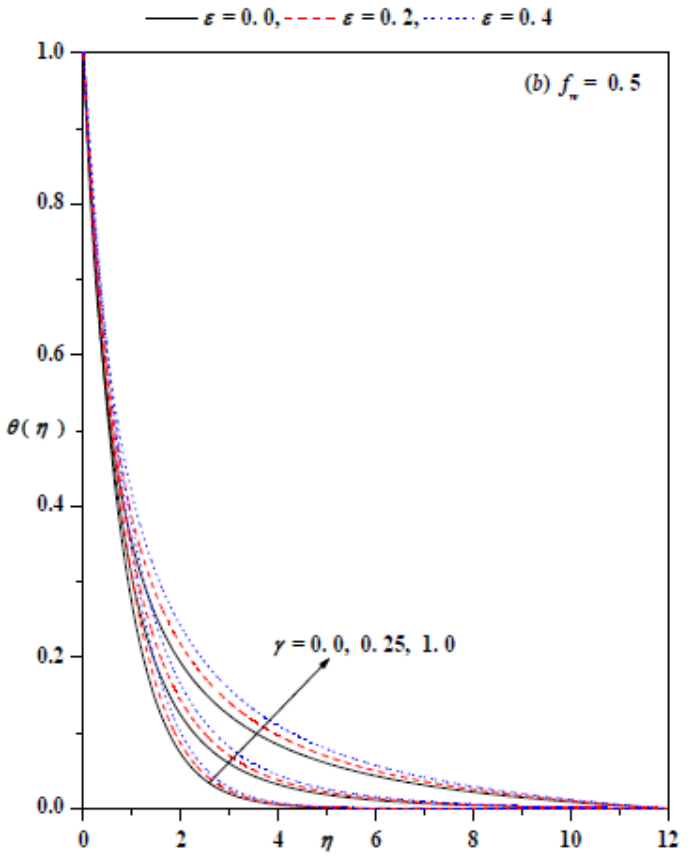

Fig 5 (b) : Temperature profile for different values of $\gamma$ and $\varepsilon$ with $\beta=-0.1, P_{r}=1.0, \theta_{r}=-5.0$ when $f_{v}=0.5$.

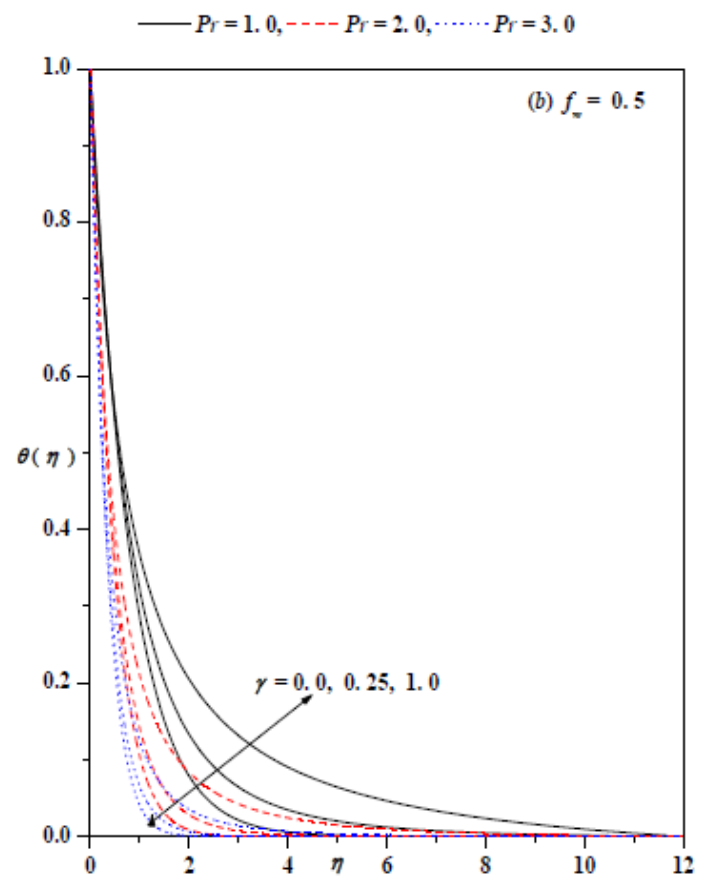

Fig 6 (b) : Temperature profile for different values of $\gamma$ and $P$ with $\beta=-0.1, \varepsilon=0.1, \theta_{r}=-5.0$ when $f_{v}=0.5$.

- In the presence of transverse curvature, the effect of the variable viscosity parameter is to decrease the velocity boundary layer thickness. However, quite the opposite is true with the thermal boundary layer thickness;

- The effect of increasing the values of the Prandtl number is to decrease the thermal boundary layer thickness and the wall temperature gradient; 
- The effects of the variable thermal conductivity and the heat source/sink parameter are to enhance the temperature; and

Of all the parameters, the variable thermo-physical property parameters have the strongest effect on the drag, heat transfer characteristics, the horizontal velocity and the temperature fields.

\section{REFERENCES}

Abel, M.S., S.K. Khan and K.V. Prasad (2002). Study of visco-elastic fluid flow and heat transfer over a stretching sheet with variable fluid viscosity. Int. J. Non-Linear Mech., 37, 81-88.

Ali, M.E. (1994). Heat transfer characteristics of a continuous stretching surface. Heat Mass Transf., 29, 227-234.

Ali, M.E. (2006). The effect of variable viscosity on mixed convection heat transfer along a vertical moving surface. Int. J. Ther. Sci., 45, 60-69.

Bachok, N. and A. Ishak (2010). Flow and heat transfer over a stretching cylinder with prescribed surface heat flux. Malaysian J. of Math. Sci., 4, 159-169.

Cebeci, T. and P. Bradshaw (1984). Physical and computational aspects of convective heat transfer. Springer-Verlag, New York.

Chen, C.K. and M.I. Char (1988). Heat transfer of a continuous stretching surface with suction or blowing. J. Math. Anal. Appl., 135, 568-580.

Cortell, R. (2005). A note on magneto hydrodynamic flow of power law fluid over a stretching sheet. Appl. Math. Comp., 168, 557-566.

Crane, L.J. (1970). Flow past a stretching plate. ZAMP, 21, 645-647.

Crane, L.J. (1975). Boundary layer flow due to a stretching cylinder. ZAMP, 26, 619-622.

Datti, P.S., K.V. Prasad, M.S. Abel and A. Joshi (2004). MHD fluid flow over a non-isothermal stretching sheet. Int. J. Eng. Sci. 42, 935.

Erickson, L.E., L.T. Fan and V.G. Fox (1966). Heat and mass transfer on a moving continuous flat plate with suction or injection. Ind. Engng. Chem. Fund., 5, 19-25.

Grubka, L.G. and K.M. Bobba (1985). Heat transfer characteristics of a continuous stretching surface with variable temperature. ASME J. Heat Transf., 107, 248-250.

Gupta, P.S. and A.S. Gupta (1977). Heat and mass transfer on a stretching sheet with suction or blowing. Can. J. Chem. Engg., 55, 744- 746.

Hassanien, I.A. (1997). The effect of variable viscosity on flow and heat transfer on a continuous stretching surface. ZAMM, 77, 876-880.

Herwig, H. and C. Wickern (1986). The effect of variable properties on laminar boundary layer flow. Wärme- und Stoffübertragung, 20, 47-57.

Ishak, A. and R. Nazar (2009). Laminar Boundary Layer Flow along a Stretching Cylinder. Euro. J. of Sci. Re., 36, 22-29.

Ishak, A., R. Nazar and I. Pop (2009). Boundary layer flow and heat transfer over an unsteady stretching vertical surface. Meccanica, 44, 369-375.

Keller, H.B. (1992). Numerical methods for two-point boundary value problems. Dover Publ., NewYork.

Lai, F.C. and F.A. Kulacki (1990). The effect of variable viscosity on convective heat transfer along a vertical surface in a saturated porous medium. Int. J. Heat Mass Transf., 33, 10281031.

Mahmood, T. and J.H. Merkin (1988). Similarity solutions in axisymmetric mixed- convection boundary-layer flow. J. Engng. Math., 22, 73-92.

Pop, I., M. Kumari and G. Nath (1990). NonNewtonian boundary-layers on a moving cylinder. Int. J. Engg. Sci., 28, 303-312.

Prasad, K.V., Dulal Pal and P.S. Datti (2009). MHD power law fluid flow and heat transfer over a nonisothermal stretching sheet. Comm. Nonlinear Sci. Numer. Simul., 14, 2178-2189.

Prasad, K.V., K. Vajravelu and P.S. Datti (2010). The effects of variable fluid properties on the hydromagnetic flow and heat transfer over a nonlinearly stretching sheet. Int. J. Ther. Sci., 49, 603610.

Rajagopal, K.R., T.Y. Na and A.S. Gupta (1984). Flow of a visco-elastic fluid over a stretching sheet. Rheol. Acta, 23, 213-215.

Vajravelu, K. (1994). Flow and heat transfer in a saturated porous medium over a stretching surface. ZAMP, 74, 605-614.

Wang, C.Y. (1988). Fluid flow due to a stretching cylinder. Phys fluids., 31, 466-468.

Weast, R.C. (1986-1987). CRC handbook of chemistry and physics. ( $6^{\text {th }}$ edition), CRC press, Boca Raton, Florida. 